\title{
Evaluación de los cuestionarios de sueño en la pesquisa de pacientes con síndrome de apneas obstructivas del sueño
}

\author{
FERNANDO SALDÍAS PEÑAFIEL ${ }^{1}$, JAVIERA GASSMANN PONIACHI- \\ $\mathrm{K}^{\mathrm{a}}$, ALEJANDRO CANELO LÓPEZ ${ }^{\mathrm{a}}$, JAVIER URIBE MONASTERIO ${ }^{1}$, \\ ORLANDO DÍAZ PATIÑO ${ }^{1}$
}

\section{Accuracy of sleep questionnaires for obstructive sleep apnea syndrome screening}

Background: Simple but accurate tools should be used to identify patients with obstructive sleep apnea syndrome (OSAS), aiming at an early detection and prevention of serious consequences. Aim: To assess the predictive value of four sleep questionnaires (Berlin, Epworth Sleepiness Scale [ESS], STOP, and STOP-Bang) in the screening of patients with OSAS. Material and Methods: The four sleep questionnaires were administered to 1,050 snorers aged $56 \pm 15$ years (68\% males) assessed at a sleep clinic. An overnight unattended respiratory polygraphy was performed to all patients to confirm the diagnosis of OSAS. The sensitivity, specificity, positive and negative predictive values of the four questionnaires were calculated. Results: Eighty four percent of participants had OSAS. The clinical variables associated with OSAS risk were age, male gender, hypertension, overweight, cervical circumference, waist/hip ratio, history of snoring, witnessed apneas and nycturia. Eighty-three, 86, 92 and $46 \%$ of cases were classified as having a high risk for OSAS, according to the Berlin, STOP and STOP-Bang questionnaires and ESS, respectively. STOP and STOP-Bang questionnaires had the highest sensitivity to predict OSAS (88 and 95\%, respectively) while the Flemons Index had the highest specificity (82\%). Conclusions: Sleep questionnaires were able to identify patients with a high risk for OSAS but without accurately excluding those at low risk.

(Rev Med Chile 2018; 146: 1123-1134)

Key words: Diagnosis; Sleep Apnea, Obstructive; Surveys and Questionnaires.

$\mathrm{E}$ 1 síndrome de apneas-hipopneas obstructivas del sueño (SAHOS) constituye un problema de salud prevalente en la población adulta, se estima que la mayoría de los pacientes no han sido diagnosticados debido a la falta de sensibilidad y especificidad de las manifestaciones clínicas, problemas de accesibilidad a los servicios de salud y elevados costos del estudio diagnóstico ${ }^{1-3}$. Los pacientes con SAHOS presentan episodios repetitivos de obstrucción de la vía aérea superior

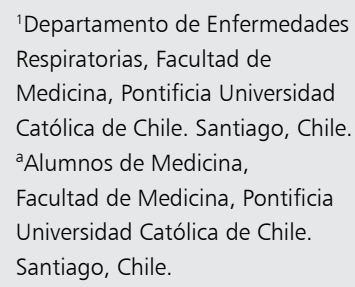

Recibido el 12 de marzo de 2018 aceptado el 21 de septiembre de 2018.

Correspondencia a:

Dr. Fernando Saldías Peñafiel Departamento de Enfermedades Respiratorias

División de Medicina, Facultad de Medicina, Pontificia Universidad Católica de Chile.

Diagonal Paraguay 362 - Sexto

Piso. Santiago, Chile.

Teléfonos: (562) 26331541 -

(562) 23543242

Fax: (562) 26335255

fsaldias@med.puc.cl durante el sueño, ocasionando episodios de hipoxemia, hipercarbia y fragmentación del sueño, lo cual determina los síntomas neurocognitivos (somnolencia diurna excesiva, disminución del estado de alerta, pérdida de memoria y capacidad de concentración, trastornos del ánimo, aumento del riesgo de accidentes laborales y del tránsito) y aumento del riesgo cardiovascular (hipertensión arterial, arritmias, eventos coronarios y accidentes cerebrovasculares isquémicos) $)^{4-7}$. 
La polisomnografía (PSG) realizada en el laboratorio de sueño es considerado el examen de referencia para confirmar el diagnóstico y establecer la gravedad del SAHOS ${ }^{8}$. Sin embargo, la necesidad de personal entrenado, la complejidad y elevado costo del examen han limitado el acceso al proceso diagnóstico de los pacientes con síntomas sugerentes de trastornos respiratorios del sueño ${ }^{3}$. Para ello, se han diseñado y validado equipos más sencillos y de menor costo de manejo ambulatorio, denominados poligrafías respiratorias (PR), que permiten establecer el diagnóstico de esta condición ${ }^{9,10}$.

Se han diseñado varias reglas predictivas, basadas en elementos de la historia clínica, examen físico y factores de riesgo, con el propósito de identificar a los pacientes con trastornos respiratorios del sueño ${ }^{11-17}$. Los cuestionarios de sueño han permitido definir la probabilidad de SAHOS en la población atendida en los servicios de salud, lo cual nos permite optimizar el uso de los recursos diagnósticos; de este modo, en los pacientes con baja probabilidad de SAHOS se recomienda solicitar una polisomnografía y en aquellos con alta probabilidad de SAHOS se puede solicitar una poligrafía respiratoria. Estas reglas predictivas han sido examinadas en distintos contextos clínicos (servicios de atención primaria, evaluación preoperatoria en servicios de cirugía, clínicas de sueño) y muestras poblacionales (población general, adultos mayores, portadores de enfermedades respiratorias, cardiovasculares y neurológicas crónicas). Las principales reglas predictivas empleadas en los servicios de atención primaria para pesquisar a los pacientes con SAHOS son el índice de Flemons ${ }^{14}$ y el cuestionario de Berlin ${ }^{18}$; mientras que en la evaluación preoperatoria se utiliza los cuestionarios STOP ${ }^{19}$ y STOP-Bang ${ }^{20}$. La escala de Epworth $^{21}$ y escala de somnolencia de Stanford ${ }^{22}$ son utilizadas para evaluar la somnolencia diurna excesiva.

El propósito del estudio es examinar el valor predictivo de los diferentes cuestionarios clínicos en la pesquisa de pacientes con síndrome de apneas obstructivas del sueño en una cohorte de sujetos roncadores atendidos en una clínica de sueño.

\section{Pacientes y Métodos}

Estudio clínico descriptivo prospectivo observacional que examinó el valor predictivo de los cuestionarios clínicos en la pesquisa de SAHOS en pacientes adultos roncadores atendidos en el Programa de Trastornos Respiratorios del Sueño del Laboratorio de Función Pulmonar de la Red de Salud UC Christus en el período de 1 de enero de 2015 hasta el 31 de diciembre de 2017. El protocolo fue revisado y aprobado por el Comité de Ética en Investigación de la Facultad de Medicina de la Institución. Los pacientes respondieron a una entrevista clínica estandarizada de sueño, los cuestionarios de sueño autoadministrados (cuestionarios de Berlin ${ }^{18}$, STOP ${ }^{19}$ y STOP-Bang ${ }^{20}$ ), la escala de Thornton, escala de Epworth ${ }^{21}$, escala de somnolencia de Stanford ${ }^{22}$ y el inventario de depresión de Beck ${ }^{23}$. A todos los pacientes se les realizó una poligrafía respiratoria en el domicilio para confirmar o descartar el diagnóstico de SAHOS. Se excluyeron del estudio a los pacientes: a) Menores de 18 años; b) Comorbilidades descompensadas; c) Diagnóstico previo de SAHOS; d) Enfermedades cardiovasculares, respiratorias, metabólicas o neurológicas crónicas en etapa avanzada; e) Incapacidad de responder a los cuestionarios clínicos o realizar el estudio de sueño.

En la evaluación clínica estandarizada realizada a los pacientes atendidos en el programa de trastornos respiratorios del sueño se consignaron la edad, género, ocupación, comorbilidades, uso de medicamentos, consumo de tabaco y alcohol, peso, talla, índice de masa corporal (IMC), perímetro cervical, circunferencia de cintura y cadera, historia de sueño: duración, presencia de ronquido, pausas respiratorias, insomnio, cefalea matinal, nicturia, inquietud psicomotora nocturna, sofocación nocturna, sueño poco reparador; el índice de Flemons ${ }^{14}$, cuestionarios de sueño de Berlin, STOP y STOP-Bang, la escala de Thornton, inventario de depresión de Beck y la magnitud de la somnolencia diurna fue evaluada con la escala de Epworth y escala de somnolencia de Stanford.

\section{Cuestionarios de sueño}

\section{Cuestionario de Berlín}

El cuestionario permite estimar el riesgo de presentar trastornos respiratorios del sueño (SAHOS) en la población adulta atendida en los servicios de atención primaria ${ }^{18}$. En el diseño del instrumento, se identificaron los principales factores de riesgo asociados al SAHOS en la población y luego se diseñaron las preguntas, 
tomando en consideración los factores de riesgo más importantes. Las preguntas del cuestionario se enfocaron en los siguientes aspectos: la presencia de ronquido, pausas respiratorias, somnolencia diurna, obesidad e hipertensión arterial. La primera categoría comprende cinco preguntas sobre el ronquido y apneas presenciadas. La segunda categoría comprende cuatro preguntas sobre la somnolencia diurna. La tercera categoría indaga acerca del antecedente de hipertensión arterial (PA $>140 / 90 \mathrm{mmHg}$ ) y obesidad (IMC $>30 \mathrm{~kg} /$ $\mathrm{m}^{2}$ ). Un paciente se considera que tiene riesgo alto de presentar SAHOS cuando posee dos o más categorías positivas o de lo contrario se considera que tiene riesgo bajo de presentar SAHOS.

\section{Cuestionarios STOP y STOP-Bang}

El cuestionario STOP consiste en cuatro preguntas que evalúan la presencia de ronquido intenso, somnolencia diurna o fatigabilidad, apneas presenciadas durante el sueño e hipertensión arterial ${ }^{19}$. En el modelo extendido, STOP-Bang, se incorporan cuatro parámetros adicionales: edad, género, índice de masa corporal y circunferencia cervical ${ }^{20}$. Las respuestas a las preguntas de ambos cuestionarios fueron diseñadas en un formato simple Si/No y las puntuaciones fluctúan entre 0 y 4 u 8 puntos, respectivamente. Se identifica a los sujetos con riesgo elevado de SAHOS cuando se responden afirmativamente al menos dos (STOP) o tres (STOP-Bang) preguntas ${ }^{19,20,24}$.

\section{Escala de somnolencia de Epworth}

Cuestionario autoadministrado que solicita a los pacientes que califiquen la probabilidad de quedarse dormido en ocho situaciones o actividades específicas de la vida cotidiana ${ }^{21}$. Se califica en una escala de 0 a 3 puntos cada situación y la puntuación puede oscilar entre 0 y 24 . A mayor puntaje, mayor será el nivel de somnolencia diurna de la persona. Se considera normal un puntaje entre 0 y 10 , y somnolencia diurna excesiva entre 11 y 24. La puntuación de la escala de Epworth se clasifica de bajo riesgo de somnolencia (menor de 11 puntos) y alto riesgo de somnolencia (11-24 puntos).

\section{Escala de somnolencia de Stanford}

El cuestionario mide los niveles de somnolencia subjetiva en el momento de la evaluación, se compone de siete descripciones de estados pro- gresivos de somnolencia, entre las cuales el sujeto debe elegir la que indica su nivel de somnolencia actual $^{22}$. El resultado se expresa entre 1 y 7 , un puntaje mayor o igual a 3 se asocia con una disminución en el rendimiento que está relacionado con la somnolencia.

\section{Estudio trastornos respiratorios del sueño}

La medición y cuantificación de los trastornos respiratorios del sueño se realizó en el domicilio del paciente con un equipo de poligrafía respiratoria marca Embletta Gold o Embletta MPR (Natus Neurology Incorporated, Middleton, WI, USA) que cumple con las exigencias de la Academia Americana de Medicina del Sueño (AASM) para los estudios de nivel $\mathrm{III}^{9,10,25}$, el cual mide el flujo aéreo oronasal, esfuerzo respiratorio torácico y abdominal, pulsioximetría, posición corporal y ronquido. El análisis de la PR fue realizado manualmente por un médico especialista en enfermedades respiratorias que desconocía los antecedentes clínicos del paciente y los resultados de los cuestionarios de sueño; consignando la duración del estudio, posición corporal, número de apneas e hipopneas obstructivas, centrales y mixtas, caída de la saturación arterial de oxígeno bajo 90\% (CT90\%) y número de episodios de desaturación mayor de $4 \%$. Se calculó el número de eventos respiratorios dividiendo el número total de apneas e hipopneas por el tiempo total de registro en horas. La ejecución e interpretación de los exámenes siguió las recomendaciones de la Academia Americana de Medicina del Sueño ${ }^{26}$. El diagnóstico y la gravedad del SAHOS se clasificó siguiendo las recomendaciones internacionales: registro normal $(\mathrm{IAH}<5$ eventos/h), SAHOS leve (IAH: 5-14,9), moderado (IAH: 15-29,9) y grave (IAH $\geq 30$ eventos/h).

\section{Análisis estadístico}

Los resultados fueron expresados como valores promedio \pm desviación estándar para las variables numéricas de distribución normal y en porcentaje para las medidas en escala nominal. Las variables de distribución desconocida fueron consignadas como mediana y rangos intercuartílicos. Las variables cualitativas fueron comparadas mediante la prueba de chi cuadrado y el test exacto de Fisher, y las variables continuas según su distribución con la 
Pesquisa de pacientes con síndrome de apneas obstructivas del sueño - F. Saldías Peñafiel et al

prueba t de Student o la prueba de Mann-Whitney. Para ello se utilizaron los programas estadísticos SPSS 22.0 (SPSS Inc, Chicago) y Epi-Info 7.0 (CDC-Atlanta). Las variables clínicas y las reglas predictivas asociadas al riesgo de SAHOS fueron sometidas a análisis univariado y multivariado en un modelo de regresión logística (modalidad stepwise) que permite el control simultáneo de múltiples factores. De este modo, los parámetros que no agregaron valor predictivo no fueron

Tabla 1. Características clínicas de los pacientes atendidos en el programa de trastornos respiratorios del sueño

\begin{tabular}{|c|c|c|}
\hline Características & n $(X \pm D E)$ & Porcentaje (Rango) \\
\hline $\mathrm{n}$ & 1.050 & \\
\hline Edad (años) & $55,5 \pm 14,6$ & $18-92$ \\
\hline Género (Masculino-Femenino) & $718-332$ & $68,4-31,6$ \\
\hline Actividad laboral & $949 / 1021$ & 92,9 \\
\hline Consumo de tabaco: No fuma-Exfumador-Fumador activo & $566-300-184$ & $53,9-28,6-17,5$ \\
\hline Comorbilidades & 971 & 92,5 \\
\hline Cardiopatía coronaria & 105 & 10,0 \\
\hline Hipertensión arterial & 551 & 52,5 \\
\hline Diabetes mellitus & 268 & 25,5 \\
\hline Dislipidemia & 274 & 26,1 \\
\hline Rinitis alérgica & 324 & 30,9 \\
\hline Asma-EPOC & $101-65$ & $9,6-6,2$ \\
\hline Reflujo gastroesofágico & 435 & 41,4 \\
\hline Depresión & 226 & 21,5 \\
\hline Hipotiroidismo & 168 & 16,0 \\
\hline Peso (Kg) & $87,7 \pm 17,8$ & $32-200$ \\
\hline Talla (cm) & $167,4 \pm 9,5$ & $130-191$ \\
\hline IMC $\left(\mathrm{Kg} / \mathrm{m}^{2}\right)$ & $31,3 \pm 5,7$ & $16,7-58,5$ \\
\hline Perímetro cervical (cm) & $41,6 \pm 4,4$ & $27-58$ \\
\hline Circunferencia de cintura $(\mathrm{cm})$ & $108,1 \pm 13,6$ & $61-166$ \\
\hline Circunferencia de cadera $(\mathrm{cm})$ & $109,8 \pm 10,9$ & $83-166$ \\
\hline Índice cintura/cadera & $0,98 \pm 0,08$ & $0,58-1,41$ \\
\hline Presión arterial sistólica (mmHg) & $126,9 \pm 19,8$ & $81-230$ \\
\hline Presión arterial diastólica $(\mathrm{mmHg})$ & $80,1 \pm 13,1$ & $50-152$ \\
\hline Escala de Mallampati: I-II-III-IV & $61-144-188-621$ & $6,0-14,2-18,5-61,2$ \\
\hline Roncador habitual & 923 & 87,9 \\
\hline Apneas presenciadas & 820 & 78,1 \\
\hline Somnolencia diurna excesiva & 950 & 90,5 \\
\hline Sueño poco reparador & 658 & 62,7 \\
\hline Insomnio & 403 & 38,4 \\
\hline Cefalea matinal & 401 & 38,2 \\
\hline Nicturia & 704 & 67,0 \\
\hline Sofocación nocturna & $126 / 316$ & 39,9 \\
\hline Inquietud psicomotora nocturna & $124 / 385$ & 32,2 \\
\hline SAHOS leve-moderado-grave & $271-231-378$ & $25,8-22,0-36,0$ \\
\hline
\end{tabular}

Nota: $X \pm D E$ : Promedio \pm Desviación estándar; EPOC: Enfermedad Pulmonar Obstructiva Crónica; IMC: Índice de masa corporal; SAHOS: Síndrome de apneas-hipopneas obstructivas del sueño. 
retenidos en el modelo. Se midió la sensibilidad, especificidad, valor predictivo positivo y negativo y las razones de probabilidades (odds ratio) de los índices predictores de SAHOS, considerando los puntos de corte definidos por los autores ${ }^{14,18-22}$. Para comparar la sensibilidad y especificidad de las distintas reglas predictoras se utilizó el test de $\mathrm{McNemar}$. Las curvas receptor operador (ROC) de los modelos predictivos nos permitió comparar su capacidad para predecir el diagnóstico de SAHOS. Para ello, se consignó el área bajo la curva ROC, el intervalo de confianza de $95 \%$ y valor de p. Las diferencias entre las variables fueron consideradas significativas con un valor de $\mathrm{p}<0,05$.

\section{Resultados}

Se evaluaron 1.050 pacientes roncadores en el programa de trastornos respiratorios del sueño de la Red de Salud UC Christus, edad: $56 \pm 15$ años (rango: 18-92), 68\% varones, 93\% eran trabajadores activos, $46 \%$ fumadores o exfumadores, 93\% tienen enfermedades crónicas, especialmente hipertensión arterial, diabetes mellitus, dislipidemia, rinitis alérgica, reflujo gastroesofágico y depresión (Tabla 1). La mayoría de los pacientes refieren ronquido intenso de larga data, apneas presenciadas, somnolencia diurna, nicturia, sueño poco reparador, cefalea matinal, insomnio, sofocación e inquietud psicomotora nocturna. En esta cohorte, $84 \%$ presentaba síndrome de apneas obstructivas del sueño, siendo dos tercios de magnitud moderada o severa. En la Tabla 2 se describen los principales factores de riesgo y cuestionarios de sueño predictores de SAHOS de esta cohorte.

Tabla 2. Factores de riesgo y cuestionarios de sueño de los pacientes atendidos en el programa de trastornos respiratorios del sueño

\begin{tabular}{|c|c|c|}
\hline Características & n $(X \pm D E)$ & Porcentaje (Rango) \\
\hline $\mathrm{n}$ & 1.050 & \\
\hline Duración del sueño (horas) & $7,34 \pm 1,61$ & $3-13$ \\
\hline $\mathrm{IMC}>30 \mathrm{~kg} / \mathrm{m}^{2}$ & $562 / 1028$ & 54,7 \\
\hline Perímetro cervical $>40 \mathrm{~cm}$ & $611 / 971$ & 62,9 \\
\hline Índice cintura/cadera $>0,94$ & $678 / 911$ & 74,4 \\
\hline Cuestionario de depresión de Beck sugerente de trastorno del ánimo & $277 / 1014$ & 27,3 \\
\hline Índice de Flemons & $46,4 \pm 5,4$ & $30-62$ \\
\hline Índice de Flemons: Alto riesgo de SAHOS & $366 / 1017$ & 36,0 \\
\hline Cuestionario de Berlin: Alto riesgo de SAHOS & $251 / 302$ & 83,1 \\
\hline STOP: Alto riesgo de SAHOS & $898 / 1050$ & 85,5 \\
\hline STOP-Bang: Alto riesgo de SAHOS & $969 / 1050$ & 92,3 \\
\hline Escala de Epworth & $10,5 \pm 5,5$ & $0-24$ \\
\hline Escala de Epworth: Somnolencia diurna excesiva & $473 / 1032$ & 45,8 \\
\hline Accidentes de tránsito & $37 / 996$ & 3,7 \\
\hline Escala de Stanford: Somnolencia diurna excesiva & $460 / 880$ & 52,3 \\
\hline $\mathrm{SaO}_{2}$ basal (\%) & $95,8 \pm 2,2$ & $80-99$ \\
\hline $\mathrm{SaO}_{2}$ media (\%) & $92,3 \pm 3,6$ & $60-98$ \\
\hline $\mathrm{SaO}_{2}$ mínima (\%) & $78,6 \pm 9,8$ & $50-95$ \\
\hline CT-90\% (\%) & $17,8 \pm 25,8$ & $0-100$ \\
\hline ID4\% & $25,0 \pm 22,4$ & $0-118$ \\
\hline Frecuencia cardiaca media (lat/min) & $66,3 \pm 9,7$ & $40-111$ \\
\hline Índice de apneas-hipopneas & $26,0 \pm 22,4$ & $0-121$ \\
\hline
\end{tabular}

Nota: X \pm DE: Promedio \pm Desviación estándar; IMC: Índice de masa corporal; SAHOS: Síndrome de apneas-hipopneas obstructivas del sueño; $\mathrm{SaO}_{2}$ : Saturación arterial de oxígeno; CT90\%: Porcentaje del tiempo con SaO bajo 90\%; ID4\%: Índice de desaturación del $4 \%$. 
Las principales variables clínicas asociadas al diagnóstico de SAHOS fueran la edad, sexo masculino, hipertensión arterial, IMC, perímetro cervical, índice cintura/cadera, apneas presenciadas por testigos y nicturia (Tablas 3 y 4). En el análisis multivariado, los principales predictores clínicos de SAHOS fueron la edad, género masculino, IMC, apneas presenciadas, nicturia y presión arterial diastólica; agregándose en los pacientes con SAHOS grave el perímetro cervical y el índice

Tabla 3. Características clínicas y factores de riesgo de los pacientes adultos portadores de SAHOS atendidos en el programa de trastornos respiratorios del sueño

\begin{tabular}{|c|c|c|c|}
\hline Características & SAHOS (-) & SAHOS (+) & $\mathbf{p}$ \\
\hline $\mathrm{n}$ & 170 & 880 & \\
\hline Edad (años) & $51,1 \pm 17,9$ & $56,4 \pm 13,7$ & $<0,0001$ \\
\hline Género (Masculino-Femenino) & $88-82$ & $630-250$ & $<0,0001$ \\
\hline Consumo de tabaco: No fuma-Exfumador-Fumador activo & $93-46-31$ & $472-254-153$ & 0,8821 \\
\hline Comorbilidades & 152 & 819 & 0,0982 \\
\hline Cardiopatía coronaria & 10 & 95 & 0,0507 \\
\hline Hipertensión arterial & 69 & 482 & 0,0007 \\
\hline Dislipidemia & 53 & 221 & 0,0995 \\
\hline Rinitis alérgica & 54 & 270 & 0,7797 \\
\hline Asma-EPOC & $15-15$ & $86-50$ & $>0,100$ \\
\hline Reflujo gastroesofágico & 71 & 364 & 0,9226 \\
\hline Diabetes mellitus & 38 & 230 & 0,3005 \\
\hline Depresión & 41 & 185 & 0,3690 \\
\hline Hipotiroidismo & 29 & 139 & 0,6810 \\
\hline $\operatorname{IMC}\left(\mathrm{Kg} / \mathrm{m}^{2}\right)$ & $28,9 \pm 5,5$ & $31,8 \pm 5,7$ & $<0,0001$ \\
\hline Perímetro cervical (cm) & $38,8 \pm 4,2$ & $42,1 \pm 4,2$ & $<0,0001$ \\
\hline Índice cintura/cadera & $0,95 \pm 0,08$ & $0,99 \pm 0,08$ & $<0,0001$ \\
\hline Presión arterial sistólica (mmHg) & $120,9 \pm 17,4$ & $128,2 \pm 20,0$ & $<0,0001$ \\
\hline Presión arterial diastólica (mmHg) & $74,8 \pm 10,1$ & $81,2 \pm 13,4$ & $<0,0001$ \\
\hline Escala de Mallampati: III-IV & $34-89$ & $154-532$ & 0,1336 \\
\hline Roncador habitual & 150 & 773 & 0,8853 \\
\hline Apneas presenciadas & 109 & 711 & $<0,0001$ \\
\hline Somnolencia diurna excesiva & 153 & 797 & 0,8174 \\
\hline Sueño poco reparador & 112 & 546 & 0,3439 \\
\hline Insomnio & 89 & 314 & $<0,0001$ \\
\hline Cefalea matinal & 71 & 330 & 0,2950 \\
\hline Nicturia & 94 & 610 & 0,0004 \\
\hline Sofocación nocturna & $23 / 78$ & $103 / 238$ & 0,0311 \\
\hline Inquietud psicomotora nocturna & $30 / 81$ & $94 / 304$ & 0,2958 \\
\hline Cuestionario de depresión de Beck & $13,6 \pm 9,3$ & $12,4 \pm 8,4$ & 0,1002 \\
\hline Índice de Flemons & $43,0 \pm 5,6$ & $47,1 \pm 5,0$ & $<0,0001$ \\
\hline Escala de Epworth & $9,7 \pm 5,5$ & $10,6 \pm 5,5$ & 0,0531 \\
\hline Escala de Thornton & $12,1 \pm 6,1$ & $14,1 \pm 6,5$ & 0,0003 \\
\hline Índice de apneas-hipopneas & $2,5 \pm 1,4$ & $30,5 \pm 21,6$ & $<0,0001$ \\
\hline
\end{tabular}

Nota: EPOC: Enfermedad Pulmonar Obstructiva Crónica; IMC: Índice de masa corporal; SAHOS: Síndrome de apneas-hipopneas obstructivas del sueño. 
cintura/cadera (Tabla 5).

El índice de Flemons, cuestionario de Berlin, STOP, STOP-Bang y la escala de Thornton permiten predecir el riesgo de SAHOS en la población atendida en la clínica de sueño (Tabla 6). La escala de somnolencia de Epworth se asoció al riesgo de SAHOS grave. Los cuestionarios STOP y STOPBang fueron más sensibles y el índice de Flemons fue más específico en la pesquisa de pacientes con SAHOS (Tabla 7).

Tabla 4. Características clínicas y factores de riesgo de los pacientes adultos portadores de SAHOS grave atendidos en el programa de trastornos respiratorios del sueño

\begin{tabular}{|c|c|c|c|}
\hline Características & SAHOS (-) & SAHOS grave (+) & $\mathbf{p}$ \\
\hline $\mathrm{n}$ & 170 & 378 & \\
\hline Edad (años) & $51,1 \pm 17,9$ & $57,2 \pm 13,7$ & $<0,0001$ \\
\hline Género (Masculino-Femenino) & $88-82$ & $302-76$ & $<0,0001$ \\
\hline Consumo de tabaco: No fuma-Exfumador-Fumador activo & $93-46-31$ & $193-111-73$ & 0,7457 \\
\hline Comorbilidades & 152 & 357 & 0,0342 \\
\hline Cardiopatía coronaria & 10 & 50 & 0,0109 \\
\hline Hipertensión arterial & 69 & 225 & $<0,0001$ \\
\hline Dislipidemia & 53 & 101 & 0,2834 \\
\hline Rinitis alérgica & 54 & 106 & 0,3758 \\
\hline Asma-EPOC & $15-15$ & -19 & $>0,08$ \\
\hline Reflujo gastroesofágico & 71 & 154 & 0,8218 \\
\hline Diabetes mellitus & 38 & 114 & 0,0593 \\
\hline Depresión & 41 & 70 & 0,1317 \\
\hline Hipotiroidismo & 29 & 55 & 0,4512 \\
\hline $\mathrm{IMC}\left(\mathrm{Kg} / \mathrm{m}^{2}\right)$ & $28,9 \pm 5,5$ & $32,7 \pm 5,6$ & $<0,0001$ \\
\hline Perímetro cervical (cm) & $38,8 \pm 4,2$ & $43,7 \pm 4,0$ & $<0,0001$ \\
\hline Índice cintura/cadera & $0,95 \pm 0,08$ & $1,01 \pm 0,08$ & $<0,0001$ \\
\hline Presión arterial sistólica (mmHg) & $120,9 \pm 17,4$ & $130,6 \pm 21,1$ & $<0,0001$ \\
\hline Presión arterial diastólica $(\mathrm{mmHg})$ & $74,8 \pm 10,1$ & $83,6 \pm 14,5$ & $<0,0001$ \\
\hline Escala de Mallampati: III-IV & $34-89$ & $71-233$ & 0,0717 \\
\hline Roncador habitual & 150 & 315 & 0,1390 \\
\hline Apneas presenciadas & 109 & 319 & $<0,0001$ \\
\hline Somnolencia diurna excesiva & 153 & 347 & 0,4912 \\
\hline Sueño poco reparador & 112 & 234 & 0,3724 \\
\hline Insomnio & 89 & 125 & $<0,0001$ \\
\hline Cefalea matinal & 71 & 128 & 0,0754 \\
\hline Nicturia & 94 & 283 & $<0,0001$ \\
\hline Sofocación nocturna & $23 / 78$ & $37 / 84$ & 0,0559 \\
\hline Inquietud psicomotora nocturna & $30 / 81$ & $37 / 117$ & 0,4298 \\
\hline Cuestionario de depresión de Beck & $13,6 \pm 9,3$ & $12,3 \pm 8,3$ & 0,1108 \\
\hline Índice de Flemons & $43,0 \pm 5,6$ & $47,1 \pm 5,0$ & $<0,0001$ \\
\hline Escala de Epworth & $9,7 \pm 5,5$ & $11,6 \pm 5,5$ & 0,0002 \\
\hline Escala de Thornton & $12,1 \pm 6,1$ & $15,1 \pm 6,3$ & $<0,0001$ \\
\hline Índice de apneas-hipopneas & $2,5 \pm 1,4$ & $50,8 \pm 17,5$ & $<0,0001$ \\
\hline
\end{tabular}

Nota: EPOC: Enfermedad Pulmonar Obstructiva Crónica; IMC: Índice de masa corporal; SAHOS: Síndrome de apneas-hipopneas obstructivas del sueño. 
Tabla 5. Variables clínicas asociadas al riesgo de síndrome de apneas obstructivas del sueño. Análisis multivariado

\begin{tabular}{|lccccc|}
\hline Riesgo de SAHOS & Coeficiente & Error estándar & Odds ratio & IC95\% & p \\
\hline Edad & 0,0297 & 0,0078 & 1,03 & $1,01-1,04$ & 0,0001 \\
\hline Sexo masculino & 0,9266 & 0,2397 & 2,50 & $1,59-4,17$ & 0,0001 \\
\hline IMC & 0,0871 & 0,0211 & 1,09 & $1,05-1,13$ & 0,0001 \\
\hline Apneas & 0,8379 & 0,2579 & 2,33 & $1,41-3,84$ & 0,0012 \\
\hline Insomnio & $-0,8046$ & 0,2265 & 0,44 & $0,29-0,69$ & 0,0004 \\
\hline Nicturia & 0,4672 & 0,2374 & 1,61 & $1,01-2,56$ & 0,0491 \\
\hline PA Diastólica & 0,0329 & 0,0094 & 1,03 & $1,02-1,05$ & 0,0005 \\
\hline Riesgo de SAHOS grave & Coeficiente & Error estándar & Odds ratio & IC95\% & p \\
\hline Edad & 0,0211 & 0,0069 & 1,02 & $1,01-1,03$ & 0,0023 \\
\hline Perímetro cervical & 0,1371 & 0,0276 & 1,15 & $1,08-1,21$ & 0,0001 \\
\hline I. cintura/cadera & 3,0258 & 1,3960 & 20,61 & $1,34-317,9$ & 0,0302 \\
\hline PA Diastólica & 0,0174 & 0,0071 & 1,02 & $1,01-1,03$ & 0,0149 \\
\hline Apneas & 0,5522 & 0,2790 & 1,72 & $1,01-3,03$ & 0,0479 \\
\hline Cuestionario Beck & $-0,0419$ & 0,0130 & 0,96 & $0,93-0,98$ & 0,0013 \\
\hline Escala Epworth & 0,0599 & 0,0184 & 1,06 & $1,02-1,10$ & 0,0012 \\
\hline Escala Thornton & 0,0363 & 0,0168 & 1,04 & $1,01-1,07$ & 0,0314 \\
\hline
\end{tabular}

Tabla 6. Evaluación del área bajo la curva receptor operador (AUC) de los predictores clínicos y cuestionarios de sueño en el diagnóstico de trastornos respiratorios del sueño (SAHOS)

\begin{tabular}{|lll|}
\hline Predictores clínicos & \multicolumn{1}{c|}{$\begin{array}{c}\text { SAHOS } \\
\text { AUC (IC95\%, p) }\end{array}$} & \multicolumn{1}{c|}{$\begin{array}{c}\text { SAHOS grave } \\
\text { AUC (IC95\%, p) }\end{array}$} \\
\hline Edad & $0,59(0,56-0,62, p: 0,0005)$ & $0,55(0,52-0,58, p: 0,0072)$ \\
Índice de masa corporal & $0,65(0,62-0,68, p<0,0001)$ & $0,62(0,59-0,65, p<0,0001)$ \\
\hline Perímetro cervical & $0,70(0,67-0,73, p<0,0001)$ & $0,71(0,68-0,74, p<0,0001)$ \\
\hline Índice cintura/cadera & $0,64(0,61-0,67, p<0,0001)$ & $0,65(0,62-0,68, p<0,0001)$ \\
\hline Índice de Flemons & $0,70(0,67-0,73, p<0,0001)$ & $0,68(0,66-0,71, p<0,0001)$ \\
\hline Cuestionario de Berlin & $0,61(0,55-0,66, p: 0,0039)$ & $0,59(0,53-0,64, p: 0,0132)$ \\
\hline STOP & $0,61(0,58-0,64, p<0,0001)$ & $0,60(0,57-0,63, p<0,0001)$ \\
\hline STOP-Bang & $0,69(0,66-0,72, p<0,0001)$ & $0,68(0,65-0,70, p<0,0001)$ \\
\hline Escala de Epworth & $0,54(0,51-0,57, p: 0,077)$ & $0,59(0,56-0,62, p<0,0001)$ \\
\hline Escala de Stanford & $0,54(0,50-0,57, p: 0,157)$ & $0,51(0,48-0,54, p: 0,535)$ \\
\hline Escala de Thornton & $0,59(0,55-0,62, p: 0,0003)$ & $0,59(0,56-0,62, p<0,0001)$ \\
\hline
\end{tabular}

Nota: SAHOS: Síndrome de apneas hipopneas obstructivas del sueño, AUC: Área bajo la curva receptor operador, IC95\%: Intervalo de confianza de $95 \%$. 
Tabla 7. Sensibilidad, especificidad, valores predictivos y odds ratio de las categorías de alto riesgo de los cuestionarios de sueño en el diagnóstico de trastornos respiratorios del sueño (SAHOS)

\begin{tabular}{|lccccccc|}
\hline Cuestionarios & Sensibilidad & Especificidad & VPP & VPN & Odds ratio & IC95\% & p \\
Índice de Flemons & 0,40 & 0,82 & 0,92 & 0,21 & 2,91 & $1,92-4,39$ & $<0,0001$ \\
Berlin & 0,85 & 0,23 & 0,77 & 0,33 & 1,66 & $0,87-3,19$ & 0,1259 \\
STOP & 0,88 & 0,28 & 0,86 & 0,31 & 2,82 & $1,90-4,18$ & $<0,0001$ \\
STOP-Bang & 0,95 & 0,21 & 0,86 & 0,44 & 4,99 & $3,10-8,01$ & $<0,0001$ \\
Epworth & 0,46 & 0,57 & 0,85 & 0,17 & 1,14 & $0,82-1,59$ & 0,4412 \\
Thornton & 0,59 & 0,56 & 0,87 & 0,21 & 1,82 & $1,30-2,55$ & 0,0005 \\
\hline
\end{tabular}

Nota: VPP: Valor predictivo positivo, VPN: Valor predictivo negativo, IC95\%: Intervalo de confianza de $95 \%$.

Tabla 8. Variables clínicas y factores de riesgo incluidos en los cuestionarios de sueño utilizados para la pesquisa de pacientes con síndrome de apneas-hipopneas obstructivas del sueño ${ }^{27}$

\begin{tabular}{|c|c|c|c|c|c|c|c|c|c|}
\hline Cuestionario & Edad & Género & HTA & IMC & PC & Ronquido & Apneas & Somnolencia & Otros \\
\hline Berlin & & & $x$ & & & $x$ & $x$ & $x$ & \\
\hline STOP & & & $x$ & & & $x$ & $x$ & $x$ & \\
\hline STOP-Bang & $x$ & $x$ & $x$ & $x$ & $x$ & $x$ & $x$ & $x$ & \\
\hline Flemons & & & $x$ & & $x$ & $x$ & & & $x^{a}$ \\
\hline ASA & & & & $x$ & $x$ & $x$ & $x$ & $x$ & $X^{b}$ \\
\hline SA-SDQ & $x$ & & & $x$ & & $x$ & $x$ & & $x^{c}$ \\
\hline Wisconsin & & & & & & $x$ & $x$ & & \\
\hline
\end{tabular}

Nota: HTA: Hipertensión arterial; IMC: Índice de masa corporal; PC: Perímetro cervical; ASA: American Society of Anesthesiologists; SA-SDQ: Sleep apnea scale of the Sleep Disorders Questionnaire; a: Sofocación nocturna; b: Problemas anatómicos de la vía aérea superior; c: Sudoración nocturna y consumo de tabaco.

\section{Discusión}

Los principales hallazgos del estudio fueron: a) Una elevada proporción de los pacientes adultos atendidos en una clínica de sueño tienen SAHOS, siendo dos tercios de magnitud moderada o severa; b) Los principales predictores clínicos de SAHOS son la edad, género masculino, hipertensión arterial, índice de masa corporal, perímetro cervical, índice cintura/cadera, apneas presenciadas y nicturia; c) El índice de Flemons, cuestionario de Berlin, STOP, STOP-Bang y la escala de Thornton permiten predecir el riesgo de SAHOS; d) Los cuestionarios STOP y STOP Bang son más sensibles y el índice de Flemons es más específico en la pesquisa de pacientes con trastornos respiratorios del sueño; e) Los cuestionarios que evalúan el estado de alerta, escalas de somnolencia de Epworth y Stanford, son pobres predictores de SAHOS.
En nuestro estudio, las principales variables clínicas asociadas al riesgo de SAHOS fueron la edad, género masculino, hipertensión arterial, sobrepeso evaluado mediante el IMC, índice cintura/cadera y perímetro cervical, historia de ronquido, apneas o pausas respiratorias durante el sueño, nicturia, sueño poco reparador y somnolencia diurna excesiva. Estas variables clínicas son incluidas en los principales cuestionarios de sueño empleados en la pesquisa de pacientes con trastornos respiratorios del sueño (Tabla 8). La historia de ronquido intenso tiene elevada sensibilidad pero muy baja especificidad para la pesquisa de pacientes con SAHOS; de hecho, este síntoma lo presentan la mayoría de los pacientes derivados a las clínicas de sueño. Mientras que las apneas o pausas respiratorias presenciadas por testigos constituyen el hallazgo más específico en la historia clínica (Tablas 3 y 4). La somnolencia 
diurna excesiva objetivada mediante las escalas de somnolencia de Epworth y Stanford no nos permitió identificar a los pacientes con mayor riesgo de SAHOS, lo cual es explicable por la elevada prevalencia de este síntoma en la población general, la variable percepción del síntoma según edad y género, y su amplio diagnóstico diferencial $^{28-30}$. De este modo, las escalas de somnolencia aplicadas a sujetos roncadores no han permitido identificar con precisión a los pacientes con trastornos respiratorios del sueño ${ }^{17,31-33}$.

En el examen físico, los principales hallazgos clínicos asociados al riesgo de SAHOS fueron la pesquisa de cifras tensionales elevadas y sobrepeso objetivado mediante el IMC, perímetro cervical e índice cintura/cadera (Tablas 3-5). Similar a lo descrito en la literatura ${ }^{34-38}$, el perímetro cervical fue el principal hallazgo clínico asociado al riesgo de SAHOS (AUC: 0,70; IC95\%: 0,67-0,73, $\mathrm{p}<0,0001)$.

En nuestro estudio, los cuestionarios de Berlin, STOP y STOP-Bang, el índice de Flemons y la escala de Thornton permitieron identificar a los pacientes con riesgo elevado de SAHOS (Tabla 7). El desempeño de los cuestionarios fue variable, con áreas bajo la curva receptor operador que fluctuaron entre 0,59 y 0,70; los cuestionarios STOP y STOP-BANG fueron más sensibles y el índice de Flemons más específico en la pesquisa de pacientes con SAHOS. El cuestionario de Berlin, la escala de Epworth y el índice de Flemons han sido recomendados para la pesquisa de pacientes con trastornos respiratorios del sueño en los servicios de atención primaria ${ }^{39-42}$; mientras que los cuestionarios STOP y STOP-Bang han sido examinados y validados en pacientes sometidos a procedimientos quirúrgicos ${ }^{19,20,24}$.

En conclusión, la entrevista clínica sistemática, considerando los principales factores de riesgo de SAHOS: edad, género, antecedente de hipertensión arterial y sobrepeso, historia de ronquido intenso, apneas presenciadas, cefalea matinal, nicturia, sueño poco reparador, somnolencia diurna excesiva, y los cuestionarios de sueño diseñados y validados en diferentes poblaciones (Berlin, STOP, STOP-Bang y Flemons) permitirían identificar a los pacientes con riesgo elevado de trastornos respiratorios del sueño en los servicios de atención primaria, optimizando el uso de los procedimientos diagnósticos (polisomnografía en laboratorio de sueño y poligrafías respiratorias ambulatorias), tomando en consideración las limitaciones de acceso y costos de los exámenes. La pesquisa oportuna y el tratamiento apropiado de esta condición con equipos de presión positiva continua en la vía aérea (CPAP) han mejorado la calidad del sueño, las alteraciones neurocognitivas asociadas a la enfermedad, la calidad de vida y sobrevida a largo plazo de los pacientes, disminuyendo significativamente el riesgo cardiovascular ${ }^{43-45}$.

\section{Referencias}

1. Mannarino MR, Di Filippo F, Pirro M. Obstructive sleep apnea syndrome. Eur J Intern Med 2012; 23: 586-93.

2. Usmani ZA, Chai-Coetzer CL, Antic NA, McEvoy RD. Obstructive sleep apnoea in adults. Postgrad Med J 2013; 89: 148-56.

3. Young T, Evans L, Finn L, Palta M. Estimation of the clinically diagnosed proportion of sleep apnea syndrome in middle-aged men and women. Sleep 1997; 20: 705-6.

4. Park JG, Ramar K, Olson EJ. Updates on definition, consequences, and management of obstructive sleep apnea. Mayo Clin Proc 2011; 86: 549-54.

5. Shahar E, Whitney CW, Redline S, Lee ET, Newman $\mathrm{AB}$, Nieto FJ, et al. Sleep-disordered breathing and cardiovascular disease: cross-sectional results of the Sleep Heart Health Study. Am J Respir Crit Care Med 2001; 163: 19-25.

6. Turkington PM, Sircar M, Allgar V, Elliott MW. Relationship between obstructive sleep apnoea, driving simulator performance, and risk of road traffic accidents. Thorax 2001; 56: 800-5.

7. Yaggi HK, Concato J, Kernan WN, Lichtman JH, Brass LM, Mohsenin V. Obstructive sleep apnea as a risk factor for stroke and death. N Engl J Med 2005; 353: 2034-41.

8. Health Quality Ontario. Polysomnography in patients with obstructive sleep apnea: an evidence-based analysis. Ont Health Technol Assess Ser 2006; 6: 1-38.

9. Collop NA, Anderson WM, Boehlecke B, Claman D, Goldberg R, Gottlieb DJ, et al. Clinical guidelines for the use of unattended portable monitors in the diagnosis of obstructive sleep apnea in adult patients. Portable Monitoring Task Force of the American Academy of Sleep Medicine. J Clin Sleep Med 2007; 3: 737-47.

10. El Shayeb M, Topfer LA, Stafinski T, Pawluk L, Menon D. Diagnostic accuracy of level 3 portable sleep tests versus level 1 polysomnography for sleep-disordered breathing: a systematic review and meta-analysis. CMAJ 2014; 186: E25-51. 
11. Viner S, Szalai JP, Hoffstein V. Are history and physical examination a good screening test for sleep apnea? Ann Intern Med 1991; 115: 356-9.

12. Hoffstein V, Szalai JP. Predictive value of clinical features in diagnosing obstructive sleep apnea. Sleep 1993; 16: 118-22.

13. Kump K, Whalen C, Tishler PV, Browner I, Ferrette V, Strohl KP, et al. Assessment of the validity and utility of a sleep-symptom questionnaire. Am J Respir Crit Care Med 1994; 150: 735-41.

14. Flemons WW, Whitelaw WA, Brant R, Remmers JE. Likelihood ratios for a sleep apnea clinical prediction rule. Am J Respir Crit Care Med 1994; 150: 1279-85.

15. Deegan PC, McNicholas WT. Predictive value of clinical features for the obstructive sleep apnoea syndrome. Eur Respir J 1996; 9: 117-24.

16. Flemons WW, McNicholas WT. Clinical prediction of the sleep apnea syndrome. Sleep Med Rev 1997; 1: 1932.

17. Sil A, Barr G. Assessment of predictive ability of Epworth scoring in screening of patients with sleep apnoea. J Laryngol Otol 2012; 126: 372-9.

18. Netzer NC, Stoohs RA, Netzer CM, Clark K, Strohl KP. Using the Berlin Questionnaire to identify patients at risk for the sleep apnea syndrome. Ann Intern Med 1999; 131: 485-91.

19. Chung F, Yegneswaran B, Liao P, Chung SA, Vairavanathan S, Islam S. STOP questionnaire: a tool to screen patients for obstructive sleep apnea. Anesthesiology 2008; 108: 812-21.

20. Chung F, Abdullah HR, Liao P. STOP-Bang Questionnaire: A practical approach to screen for obstructive sleep apnea. Chest 2016; 149: 631-8.

21. Johns MW. A new method for measuring daytime sleepiness: the Epworth sleepiness scale. Sleep 1991; 14: 540-5.

22. Hoddes E, Zarcone V, Smythe H, Phillips R, Dement WC. Quantification of sleepiness: a new approach. Psychophysiology 1973; 10: 431-6.

23. Beck AT, Steer RA, Brown GK. BDI-II. Manual for the Beck Depression Inventory-Second Edition. San Antonio, TX: Psychological Corporation; 1996.

24. Nagappa M, Liao P, Wong J, Auckley D, Ramachandran SK, Memtsoudis S, et al. Validation of the STOPBang questionnaire as a screening tool for obstructive sleep apnea among different populations: A systematic review and meta-analysis. PLoS One 2015; 10: e0143697.

25. Lux L, Boehlecke B, Lohr KN. Effectiveness of Portable Monitoring Devices for Diagnosing Obstructive Sleep Apnea: Update of a Systematic Review. Rockville (MD):
Agency for Healthcare Research and Quality (US); 2004. AHRQ Technology Assessments.

26. Kapur VK, Auckley DH, Chowdhuri S, Kuhlmann DC, Mehra R, Ramar K, et al. Clinical Practice Guideline for Diagnostic Testing for Adult Obstructive Sleep Apnea: An American Academy of Sleep Medicine Clinical Practice Guideline. J Clin Sleep Med 2017; 13: 479-504.

27. Abrishami A, Khajehdehi A, Chung F. A systematic review of screening questionnaires for obstructive sleep apnea. Can J Anaesth 2010; 57: 423-38.

28. Kapur VK, Baldwin CM, Resnick HE, Gottlieb DJ, Nieto FJ. Sleepiness in patients with moderate to severe sleep-disordered breathing. Sleep 2005; 28: 472-7.

29. Roure N, Gómez S, Mediano O, Durán J, Peña M de L, Capote F, et al. Daytime sleepiness and polysomnography in obstructive sleep apnea patients. Sleep Med 2008; 9: 727-31.

30. Parkes JD. ABC of sleep disorders. Daytime sleepiness. BMJ 1993; 306: 772-5.

31. Kingshott RN, Sime PJ, Engleman HM, Douglas NJ. Self assessment of daytime sleepiness: patient versus partner. Thorax 1995; 50: 994-5.

32. Osman EZ, Osborne J, Hill PD, Lee BW. The Epworth Sleepiness Scale: can it be used for sleep apnoea screening among snorers? Clin Otolaryngol Allied Sci 1999; 24: 239-41.

33. Rosenthal LD, Dolan DC. The Epworth sleepiness scale in the identification of obstructive sleep apnea. J Nerv Ment Dis 2008; 196: 429-31.

34. Katz I, Stradling J, Slutsky AS, Zamel N, Hoffstein V. Do patients with obstructive sleep apnea have thick necks? Am Rev Respir Dis 1990; 141: 1228-31.

35. Davies RJ, Stradling JR. The relationship between neck circumference, radiographic pharyngeal anatomy, and the obstructive sleep apnea syndrome. Eur Respir J 1990; 3: 509-14.

36. Stradling JR, Crosby JH. Predictors and prevalence of obstructive sleep apnea and snoring in 1,001 middle-aged men. Thorax 1991; 46: 85-90.

37. Hoffstein V, Mateika S. Differences in abdominal and neck circumferences in patients with and without obstructive sleep apnoea. Eur Respir J 1992; 5: 377-81.

38. Davies RJ, Ali NJ, Stradling JR. Neck circumference and other clinical features in the diagnosis of the obstructive sleep apnoea syndrome. Thorax 1992; 47: 101-5.

39. Chung SA, Jairam S, Hussain MR, Shapiro CM. How, what, and why of sleep apnea. Perspectives for primary care physicians. Can Fam Physician 2002; 48: 1073-80.

40. Lieberman JA 3rd. Obstructive sleep apnea (OSA) and excessive sleepiness associated with OSA: recognition in the primary care setting. Postgrad Med 2009; 121: 33-41. 
41. Jacobs CK, Coffey J. Clinical inquiries. Sleep apnea in adults: how accurate is clinical prediction? J Fam Pract 2009; 58: 327-8.

42. Miller JN, Berger AM. Screening and assessment for obstructive sleep apnea in primary care. Sleep Med Rev 2016; 29: 41-51.

43. Lloberes P, Durán-Cantolla J, Martínez-García MA, Marín JM, Ferrer A, Corral J, et al. Diagnosis and treatment of sleep apnea-hypopnea syndrome. Spanish Society of Pulmonology and Thoracic Surgery. Arch Bronconeumol 2011; 47: 143-56.

44. Jordan AS, McSharry DG, Malhotra A. Adult obstructive sleep apnoea. Lancet 2014; 383: 736-47.

45. Lévy P, Kohler M, McNicholas WT, Barbé F, McEvoy $\mathrm{RD}$, Somers VK, et al. Obstructive sleep apnoea syndrome. Nat Rev Dis Primers 2015; 1: 15015. 